infections are the most common infections, supporting the prevention policies by vaccination for influenza viruses and Str. pneumoniae, in particular in the elderly population

REFERENCE:

[1] Ramiro S, et al. Ann Rheum Dis. 2017;76(6):1101-1136.

Disclosure of Interest: None declared

DOI: 10.1136/annrheumdis-2018-eular.2423

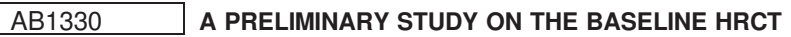 SCORING CRITERIA FOR PREDICTING THE FIBROSIS PROGRESSION OF RA-ILD}

L. Li. Department of Rheumatology and Immunology, Beijing Chaoyang Hospital West Hospital Affiliated to Capital Medical University, beijing, China

Background: Interstitial lung disease (ILD) is the most common pulmonary manifestation of RA. The progression of fibrosis in RA-ILD varied differently, and effective predictors of progression were absent.

Objectives: To explore the baseline HRCT scoring criteria that can predict progressive fibrosis and provide reference for clinical diagnosis and treatment.

Methods: The chest HRCT of RA-ILD patients from 2009 to 2017 were retrospec tively analysed, including 102 patients with progressive fibrosis and 50 patients without progressive fibrosis. Progressive fibrosis was defined as honeycombing development or an increase in extent of reticulation on follow-up HRCT. The baseline HRCT evaluation was performed by two thoracic radiologists blinded to all patient data, which including routine interstitial lesion evaluation and fibrosis predictive score. We proposed the baseline HRCT fibrosis predictive score to differentiate progressive fibrosis from the stable patients. The score included two parts, subpleural lesion score and bronchovascular bundle thickening score, with a total score of 0-9 points.

Results: In the routine evaluation, peripheral distribution was more common in the progressive fibrosis group $(78.43 \%$ VS $52 \%, p<0.05)$, traction bronchiectasis and traction bronchiolectasis both were more common in the progressive group (41.18\% VS 8\%, $\mathrm{p}<0.01 ; 93.14 \%$ VS 46\%, $\mathrm{p}<0.01$; respectively). Compared with non-progressive fibrosis group, subpleural reticulation and subpleural linear opacities were more common in the progressive fibrosis group $(79.21 \%$ VS $28 \%$, $p<0.0 ; 41.80 \%$ VS $14 \%, p<0.01$; respectively), subpleural honeycombing was more common in the progressive fibrosis group $(41.17 \%$ VS $20 \%, p<0.05)$, subpleural ground-glass opacity showed no difference between the two groups (52.94\% VS 66\%, p>0.05). Notably, subpleural honeycombing in the progressive group were all accompanied by the presence of subpleural reticulation and subpleural linear opacities on baseline HRCT. The AUC curve of fibrosis predictive score was 0.87 (95\% confidence interval, $0.81,0.93)$, indicating that baseline HRCT score had a better judgement value for progressive fibrosis. The cut-off value was 5.5 points (sensitivity was $64 \%$, specificity $94 \%$ ), that is, baseline HRCT score $>6$ points was more likely to develop progressive fibrosis. Drug interventions (glucocorticoids, cyclophosphamide) were relatively deficient in the progressive fibrosis group, (26.47\% VS $44 \%, p<0.05 ; 9.80 \%$ VS $22 \%, p<0.05$; respectively). Further analysis showed that treatment situations (glucocorticoids, cyclophosphamide) were similar between the baseline HRCT score $\geq 6$ points group and the baseline HRCT score $<6$ points group $(34.78 \%$ VS $30.12 \%$, p $>0.05 ; 10.14 \%$ VS $16.87 \%, p>0.05$; respectively), suggesting that patients with potential fibrotic tendencies had not been treated actively in the early stage.

Conclusions: The baseline HRCT score has a better predictive value for the progressive fibrosis of RA-ILD, traction bronchiectasis and traction bronchiolectasis are helpful to identify progression. The baseline HRCT evaluation may provide a reference for the choice of time for treatment.

Disclosure of Interest: None declared

DOI: 10.1136/annrheumdis-2018-eular.6103

\section{AB1331 SERUM DRUG LEVELS IN THE MANAGEMENT OF RHEUMATOID ARTHRITISAND SPONDYLOARTHRITIS: A SYSTEMATIC REVIEW}

M. Martín López ${ }^{1}$, L. Carmona ${ }^{2}$, A. Balsa ${ }^{3}$, J. Calvo-Alén $^{4}$, R. Sanmartí ${ }^{5}$ J. Tornero ${ }^{6}$, T. Otón ${ }^{2}$, J. Rosas-Gómez de Salazar ${ }^{7}{ }^{1}$ Hospital Universitario 12 de Octubre; ${ }^{2}$ Instituto de Salud Musculoesquelética (InMusc); ${ }^{3}$ Hospital Universitario La Paz, Madrid; ${ }^{4}$ Hospital Universitario Araba, Vitoria; ${ }^{5}$ Hospital Clinic de Barcelona, Barcelona; ${ }^{6}$ Hospital General Universitario de Guadalajara, Guadalajara; ${ }^{7}$ Hospital Marina Baixa, Villajoyosa-Alicante, Spain

Background: Several studies in rheumatic and other inflammatory diseases treated with tumour necrosis factor-alpha inhibitors (TNFi) support the association between clinical response and trough drug levels (DL). The utility of monitoring DL in rheumatoid arthritis (RA) and spondyloarthritis ( $\mathrm{SpA}$ ) patients on biological therapy is not clear, so it is important to identify clinical situations in which, the knowledge of $\mathrm{DL}$, would allow an individualised therapeutic decision in the patient more cost-effective.

Objectives: To answer the most relevant clinical questions related to the usefulness of DL measurement in clinical practice: 1) whether, in patients in remission who are candidates for optimisation, DL predict relapse or survival to dose-titration; 2) whether, in patients with primary or secondary failure to biological agents $\mathrm{DL}$ influence prognosis; and 3) whether concomitant use of methotrexate (MTX) influences the association between $\mathrm{DL}$ and response.

Methods: An electronic search of the major databases was performed, Medline, Embase and Cochrane Library, from inception to December 2016. We included articles published in English or Spanish on patients with RA, SpA or both, treated with TNFi, tocilizumab or abatacept, and related to answer any of the three questions of the target research. Overall characteristics and outcomes of the studies were collected in a table of evidence and the quality of the studies was assessed with a scale based on the Oxford levels of Evidence.

Results: The electronic search strategy yielded 1749 articles, twelve of which were included in this systematic review. 2 studies responded the first question, 5 the second, and 7 the third. These studies were mainly observational studies, 6 retrospective and 4 prospective cohort studies, and 2 randomised clinical trials. Sample sized varied from 24 to 395 patients, for a total of 1482 patients analysed. A total of 1281 RA patients were included (64 in the first question, 243 in the second and 1038 in the third) and $201 \mathrm{SpA}$ patients (32 in the first and second ques tions, 169 in the third). Studies were small and with limitations, but suggest that measurement of DL may be useful in patients in remission, that higher DL predict a longer relapse-free optimisation, and in patients with failure to a biological agent treatment may need individual adjustment according to the presence of $D L$ or antidrug-antibodies. In addition, MTX influences the association between response and DL in most studies, with greater controversy in tocilizumab and SpA patients. Conclusions: Monitoring DL would allow optimal use of current biological therapies, but more studies and of better quality are needed to draw definitive conclu sions. In addition, future research should be performed to determine therapeutic ranges of $\mathrm{DL}$, so that the proposed algorithms can be used in a standardised and reliable manner.

Disclosure of Interest: None declared

DOI: 10.1136/annrheumdis-2018-eular.7397

\section{AB1332 $\quad$ FACTORS ASSOCIATED TO PERSISTENCE ON GOLIMUMAB IN PATIENTS WITH INFLAMMATORY ARTHRITIS OF THE BIOBADASER REGISTRY}

M.V. Hernández ${ }^{1}$, S. Rodriguez ${ }^{1}$, R. Morlà ${ }^{1}$, C. Sánchez-Piedra ${ }^{2}$, F. SánchezAlonso $^{2}$, F.J. Manero ${ }^{3}$, B. García-Magallón ${ }^{4}$, E. Pérez-Pampín ${ }^{5}$, C. RodríguezLozano $^{6}$, M.D. Ruiz ${ }^{7}$, A. Ortiz ${ }^{8}$, C. Díaz-Torné ${ }^{9}$, L. Mateo ${ }^{10}$, J.A. Gómez-Puerta ${ }^{1}$ R. Sanmartí ${ }^{\text {, L. Cea-Calvo }}{ }^{11}$, J.J. Gómez-Reino ${ }^{5}$, on behalf of BIOBADASER Study Group. ${ }^{1}$ Rheumatology, H Clínic Barcelona, Barcelona; ${ }^{2}$ Research Unit, Spanish Society Rheumatology, Madrid; ${ }^{3}$ Rheumatology, H. Miguel Servet, Zaragoza; ${ }^{4}$ Rheumatology, H San Jorge, Huesca; ${ }^{5}$ Rheumatology, H Univ. Santiago, Santiago de Compostela; ${ }^{6}$ Rheumatology, H Gran Canaria, Las Palmas; ${ }^{7}$ Rheumatology, $H$ Virgen Macarena, Sevilla; ${ }^{8}$ Rheumatology, $H$ La Princesa, Madrid; ${ }^{9}$ Rheumatology, H Sant Pau, Barcelona; ${ }^{10}$ Rheumatology, H Germans Trias i Pujol, Badalona; ${ }^{11}$ Medical Affairs, Merck Sharp Dohme Spain, Madrid, Spain

Background: Persistence to treatment may be used as a surrogate marker for long-term treatment success

Objectives: To assess the probability of persistence on golimumab (GOL) up to 5 years after treatment initiation and the factors associated to longer persistence Methods: BIOBADASER is the Spanish registry of biological drugs of the Spanish Society of Rheumatology and the Spanish Medicines Agency. A data-base analysis was done in October 2017 on all the patients who had initiated GOL for one of the approved indications (rheumatoid arthritis [RA], axial spondyloarthritis $[\mathrm{SpA}]$ or psoriatic arthritis [PsA]). The probability of persistence was calculated with a Kaplan-Meier test. Factors related to persistence were analysed with a Cox-regression model.

Results: 353 patients were included (105 [29.8\%] RA, 147 [30.6\%] axial SpA and 101 [28.6\%] with PsA), mean age $52^{11}$ years, $55 \%$ women). Median duration of disease at the onset of GOL was 8.0 [2.8-15.0] years. GOL was the first biological drug in $40.1 \%$ of the patients, second in $30.1 \%$ and third or further biological in 29.8\%. Concomitant medication at GOL initiation was methotrexate (MTX (33.7\%), sulphasalazine (SSZ) $(6.1 \%)$, leflunomide (LEF) $(12.7 \%)$, steroids (CS) $(26.0 \%)$. At the last observation on GOL, $32.0 \%$ were on MTX, $5.8 \%$ on SSZ, $12.8 \%$ on LEF, $16.3 \%$ on CS. The probability of persistence on GOL since treatment initiation was $85.9 \%$ at year $1(95 \% \mathrm{Cl} 81.4-89.5), 73.7 \%$ at year $2(67.1-$ $79.1), 68.5 \%$ at year $3(60.5-75.1), 60.6 \%(50.2-69.5)$ at year $4 \%$ and $57.1 \%$ (44.9-67.5) at year 5. Persistence was similar for RA, axial SpA or PsA patients ( $p$ log-rank 0.070), and higher when GOL was used as first biological agent ( $p$ log rank $<0.001$ ). As first biological drug the probability of persistence was $94.5 \%$ 